\title{
Evaluation of the ability of the Clinical Treatment Score at 5 years (CTS5) compared to other risk stratification methods to predict the response to an extended endocrine therapy in breast cancer patients
}

\author{
Andrea Villasco ${ }^{1}$ (1) . Francesca Accomasso ${ }^{1} \cdot$ Marta D’Alonzo $^{1} \cdot$ Francesca Agnelli $^{2} \cdot$ Piero Sismondi $^{1}$. \\ Nicoletta Biglia ${ }^{1}$
}

Received: 23 August 2020 / Accepted: 20 April 2021 / Published online: 3 May 2021

(c) The Author(s) 2021

\begin{abstract}
Purpose Extension of adjuvant endocrine therapy (ET) reduces the risk of recurrence in women diagnosed with ER-positive breast cancers, but a significant benefit is unlikely to happen to all individual patients. This study is aimed at evaluating the ability of different clinical late distant recurrence (LDR) risk stratification methods and in particular the clinical treatment score at 5 years (CTS5) to predict the response to extended adjuvant ET.

Methods 783 patients diagnosed with ER+BC between 1988 and 2014 at Umberto I Hospital of Turin, of which 180 received an extended adjuvant ET, were retrospectively selected. They were stratified according to $\mathrm{pT}$, $\mathrm{pN}$, disease stage, tumor grade, Ki67 level, progesterone receptor status and CTS5. The primary endpoint was LDR rate. LDR rates according to ET duration were confronted in each subgroup.

Result The median duration of extended ET was 7 years (6-10). Median follow-up from diagnosis was 9 years (6-26). Retrospective risk stratification according to tumor size, nodal status, disease stage, tumor grade, Ki67 level, and progesterone receptor status did not appear to be able to predict the response to extended ET. In the CTS5 high-risk subgroup instead, the risk of developing an LDR was significantly lower in the patients who underwent extended ET compared to standard ET (HR 0.37, 95\% CI 0.15-0.91), while no significant benefit was demonstrated for low and intermediate-risk patients.

Conclusions Risk stratification according to CTS5 appeared to be predictive of the response to extended endocrine therapy in our population of real-life pre and postmenopausal patients.
\end{abstract}

Keywords Estrogen receptor-positive · Breast cancer - Late distant recurrence · CTS5 - Extended endocrine therapy

\section{Introduction}

Breast cancer is the most frequent neoplasia in women worldwide, and about $80 \%$ of new diagnoses are estrogen receptor-positive (ER+) tumors [1]. With current surgical and medical treatment, the results both in terms of disease recurrence and short-term survival for early ER+ breast cancer patients are excellent [2]. 5 years of adjuvant endocrine

Andrea Villasco

andrea.villasco@unito.it

1 Academic Division of Gynecology and Obstetrics, Mauriziano Umberto I Hospital, University of Turin, Via

Magellano 1, 10128 Turin, Italy

2 School of Medicine, University of Turin, Turin, Italy therapy (ET) reduce mortality (by 30\% with five years of Tamoxifen and $40 \%$ with an aromatase inhibitor (AI) in postmenopausal women) [3] and hence represent the gold standard treatment for these patients [4]. Nevertheless, up to 50\% of distant recurrences (DR) show up after the completion of adjuvant endocrine therapy. Numerous studies on extended ET (e-ET) have shown a significant improvement in disease outcomes, but their results are hardly applicable to the current clinical scenario. In postmenopausal women, extended therapy appears to be most beneficial in patients treated with Tamoxifen for 5 years who either continue Tamoxifen or switch to an AI-based regimen. Such schedules have now been long abandoned in favor of upfront AI therapies that have already shown superiority over Tamoxifen. On the contrary, the benefits of extending a 5-year course of adjuvant 
AI appear to be less substantial, except for specific subgroups of patients at higher risk of recurrence. For premenopausal women instead, data on extended therapy is limited and will not be applicable in the near future since the SOFT [4] and TEXT [5] trials have now revolutionized the clinical practice. On these premises, the selection of the patients at higher recurrence risk deemed to get the most benefit from an extended endocrine therapy has now become a prominent concern. Many molecular profiles have been tested and proved to provide prognostic information on LDR risk after five years of ET, even though they were created to be used at diagnosis [6]. Contrariwise, the Clinical Treatment Score at 5 years (CTS5), which integrates four clinicopathological variables, was specifically developed to estimate the LDR risk after five years of adjuvant endocrine therapy for $\mathrm{ER}+$ breast cancer. It was created and validated on the ATAC and BIG 1-98 cohorts of postmenopausal patients [7] and its prognostic ability has now been externally confirmed on different cohorts of pre and postmenopausal patients [8-11]. Apart from the prognostic information, limited data is available on the ability of these tools to predict the response to the extended ET. In particular, only the Breast Cancer Index (BCI) was shown to be predictive of the response to extended ET $[12,13]$.

This study aimed at evaluating the ability of different risk stratification methods (tumor size, nodal involvement, stage of disease, tumor grade, Ki67 level, progesterone receptor status and CTS5) carried out retrospectively to predict the response to an extended ET in a sample of pre and postmenopausal real-life patients.

\section{Materials and methods}

Between 1988 and 2014, 2806 patients were treated for breast cancer at Mauriziano Umberto I Hospital of Turin. They underwent surgery, chemotherapy, radiation therapy, and endocrine therapy according to the guidelines in force at the time.

From this data set, we selected only patients with invasive tumors, complete clinicopathological data, and full immunohistochemical characterization. Tumors were considered estrogen receptor-positive when the staining involved $>1 \%$ of the specimen cancer cells. Endocrine therapy was prescribed, according to the guidelines, to all patients with tumors fulfilling this criterion.

We retained for this analysis 783 ER-positive, HER2 negative patients after excluding 101 patients who experienced a disease recurrence during the first five years of ET and 26 patients who experienced a late locoregional recurrence without evidence of distant disease. One-hundred-eighty patients of the 783 selected underwent an e-ET. The patient selection process is outlined in Fig. 9.

The 783 remaining patients that were included in the analysis underwent a retrospective LDR stratification according to tumor size, nodal status, stage of disease, tumor grade, Ki67 level, progesterone receptor status and CTS5. CTS5 score was calculated applying the final validated formula:

$$
\begin{aligned}
\text { CTS5 }= & 0.438 \times \text { nodes }+0.988 \\
& \times\left(0.093 \times \text { size }-0.001 \times \text { size }^{2}\right. \\
& +0.375 \times \text { grade }+0.017 \times \text { age }) .
\end{aligned}
$$

Patients were categorized in three risk groups based on the score obtained: low- (CTS5 $<3.13$ ), intermediate(3.13-3.86), and high-risk (>3.86).

In each subgroup of the different risk stratifications, the patients were further divided according to endocrine therapy duration: standard 5 years course or extended regimen.

Extended endocrine therapy prescription was not the standard clinical practice in the period considered. The prescription was made according to the evaluation of the clinician of individual risk factors and in agreement with patients, with duration to be determined on the patients' tolerance to treatment.

The primary endpoint of this study was the late distant recurrence (LDR) rate.

\section{Statistical analysis}

Categorical variables were confronted using Chi-square test. Two-sided $p$ values $<0.05$ were considered statistically significant. Survival analysis was performed with the Kaplan Meier method; the differences between the curves were estimated with the log-rank test. Hazard ratios were obtained with the Cox regression model and reported at the $95 \%$ confidence interval.

\section{Results}

Out of the 783 women enrolled for this analysis, 533 (68.1\%) were postmenopausal and $250(31.9 \%)$ premenopausal. Patients' characteristics are shown in Table 1. Median follow-up after standard 5 years of adjuvant ET was 4 years (range 1-21). The $42.9 \%$ of our patients had at least a fiveyear follow up, 18.9\% a 3-4-year follow-up, the 38.2\% 2 or less years of follow-up. An Aromatase Inhibitors-based endocrine therapy was given upfront to $379(71.1 \%)$ postmenopausal women, while Tamoxifen + Ovarian Function Suppression (OFS) was the preferred choice for premenopausal women $(161 / 250,64.4 \%)$ (Table 2). Extended endocrine therapy was prescribed to 180 patients, $107 / 533$ 
Table 1 Patients' characteristics

\begin{tabular}{lll}
\hline & 5 years of ET & Extended ET \\
\hline $\begin{array}{l}\text { Total } \\
\text { Menopausal Status }\end{array}$ & $603(77.0 \%)$ & $180(23.0 \%)$ \\
$\quad \begin{array}{l}\text { Premenopausal } \\
\text { Postmenopausal }\end{array}$ & $177(70.8 \%)$ & $73(29.2 \%)$ \\
Median age & $426(79.9 \%)$ & $107(20.1 \%)$ \\
Premenopausal & $45(26-54)$ & $45(27-54)$ \\
Postmenopausal & $67(51-91)$ & $64(51-83)$ \\
Median ET duration & & $7(6-10)$ \\
Premenopausal & 5 & $7(6-10)$ \\
Postmenopausal & 5 & $114(21.2 \%)$ \\
Surgery & & $66(27.0 \%)$ \\
BCS & $425(78.8 \%)$ & $9(5 \%)$ \\
Mastectomy & $178(73.0 \%)$ & \\
LDR rate & & \\
Total events & $54(9 \%)$ & \\
\hline
\end{tabular}

(20.1\%) postmenopausal and 73/250 (29.2\%) premenopausal. For these patients, the median total duration of endocrine therapy was 7 years (6-10). Out of the 73 premenopausal patients who have prescribed an extended ET, 34 (46.6\%) switched from Tamoxifen \pm OFS to an AI, while the remaining 39 (53.4\%) continued Tamoxifen without OFS (15 patients previously on Tamoxifen alone and 24 patients previously on OFS). Concerning postmenopausal patients, 83 (77.6\%) continued on the same AI administered during the previous five years while 20 (18.7\%) switched from Tamoxifen to an AI. Only four postmenopausal patients continued Tamoxifen alone in the extended setting.

Global LDR rate was $8 \%$ : $9 \%$ in the 5 years ET group and $5 \%$ in the extended ET group $(p=0.08)$ (Table 1). Median follow-up among patients who experienced an LDR was similar in both groups (5y ET: 10 years [6-26], extended ET: 10 years [6-16]).

To identify the patients at higher risk of LDR, a retrospective stratification was carried out according to CTS5 and other parameters commonly used in the clinical practice: tumor size (T1, T2, T3 or higher), nodal involvement (pN0, pN1, pN2-3), stage of the disease (Stages I, II, III), tumor grade (low, intermediate, high), Ki67 (low [ $<20 \%]$, high $[>20 \%]$ ), and progesterone receptor status (negative $[<1 \%]$, positive [ $>1 \%]$ ). The prescription of extended endocrine therapy was significantly associated to worse clinical and pathological features. We observed a higher prescription rate in patients with larger tumors $(38.5 \%$ of pT3-4 vs $26.9 \%$ of pT2 vs $19.5 \%$ of pT1, $p=0.01)$, patients with greater nodal involvement ( $34.3 \%$ of $\mathrm{pN} 2-3$ vs $30.6 \%$ of $\mathrm{pN} 1$ vs $16.9 \%$ of $\mathrm{pN} 0, p<0.001)$, patients with a higher clinical stage (35.3\% of Stage III vs $25.9 \%$ of Stage II vs $15.5 \%$ of Stage I, $p<0.001$ ), patients with higher Ki67 (33.2\% of Ki67 $>20 \%$ vs $16.5 \%$ of $\mathrm{Ki} 67<20 \%$ ), and patients with higher histologic grade (32.6\% of G3 vs $17.8 \%$ of G2 vs $10.3 \%$ of $\mathrm{G} 1, p<0.001)$. Instead, PgR status did not appear to affect e-ET prescription rate (18.7\% of PgR-negative vs 23.4\% PgR-positive patients, $p=0.39$ ). After obtaining the CTS5 score for all patients, we observed that an extended ET was also prescribed in a significantly higher proportion to patients categorized as High-Risk $(31.9 \%$ vs $14.9 \%$ lowrisk, $p<0.001)$. Details are shown in Table 3 .

No significant difference in terms of LDR risk could be observed between patients undergoing standard versus extended ET when they were categorized according to tumor size (Fig. 1), nodal involvement (Fig. 2), stage of disease (Figs. 3, 4), tumor grade (Fig. 5), Ki67 level (Fig. 6) and progesterone receptor status (Fig. 7).

Table 2 Endocrine therapy

Extended ET

Premenopausal

$73(29.2 \%)$

Tamoxifen
Tamoxifen + OFS

Aromatase Inhibitor

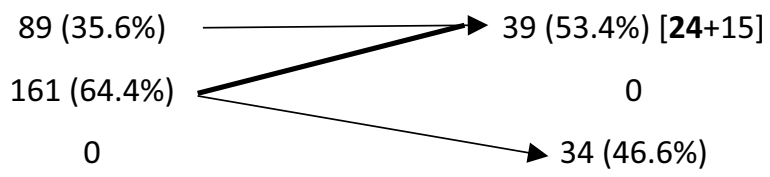

$107(20.1 \%)$

Postmenopausal

Tamoxifen
Switch
Aromatase Inhibitor

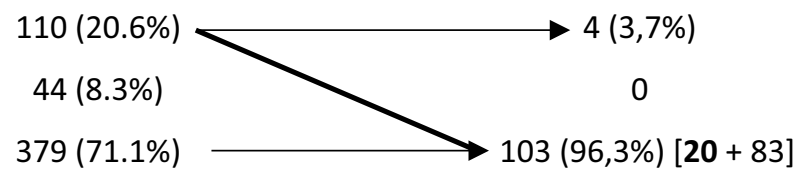

*Arrows and numbers in bold: patients switching from one type of ET to the other, detail in text 
Table 3 Extended ET according to pT, pN, Stage, ki67, PgR status and histologic tumor grade

\begin{tabular}{|c|c|c|c|}
\hline & 5 years of ET & Extended ET & $p$ value \\
\hline \multicolumn{4}{|l|}{ Tumor size } \\
\hline pT1 & $367(80.5 \%)$ & $89(19.5 \%)$ & \\
\hline pT2 & $220(73.1 \%)$ & $81(26.9 \%)$ & 0.01 \\
\hline pT3-4 & $16(61.5 \%)$ & $10(38.5 \%)$ & \\
\hline \multicolumn{4}{|l|}{ Positive nodes } \\
\hline pNO & $384(83.1 \%)$ & $78(16.9 \%)$ & $<0.001$ \\
\hline pN1 & $154(69.4 \%)$ & $68(30.6 \%)$ & \\
\hline pN2-3 & $65(65.7 \%)$ & $34(34.3 \%)$ & \\
\hline Stage & & & $<0.001$ \\
\hline Stage I & $277(84.5 \%)$ & $51(15.5 \%)$ & \\
\hline Stage II & $249(74.1 \%)$ & $87(25.9 \%)$ & \\
\hline Stage III & $77(64.7 \%)$ & $42(35.3 \%)$ & \\
\hline \multicolumn{4}{|l|}{ Ki67 } \\
\hline Low $(<20 \%)$ & $400(83.5 \%)$ & $79(16.5 \%)$ & $<0.001$ \\
\hline High $(>20 \%)$ & $203(66.8 \%)$ & $101(33.2 \%)$ & \\
\hline \multicolumn{4}{|l|}{ Histologic Grade } \\
\hline G1 & $113(89.7 \%)$ & $13(10.3 \%)$ & \\
\hline G2 & $263(82.2 \%)$ & $57(17.8 \%)$ & $<0.001$ \\
\hline G3 & $227(67.4 \%)$ & $110(32.6 \%)$ & \\
\hline \multicolumn{4}{|l|}{ PgR status } \\
\hline Negative $(<1 \%)$ & $61(81.3 \%)$ & $14(18.7 \%)$ & 0.390 \\
\hline Positive (>1\%) & $542(76.6 \%)$ & $166(23.4 \%)$ & \\
\hline \multicolumn{4}{|l|}{ CTS5 } \\
\hline Low risk & $240(85.1 \%)$ & $42(14.9 \%)$ & \\
\hline Intermediate risk & $179(77.5 \%)$ & $52(22.5 \%)$ & $<0.001$ \\
\hline High risk & $184(68.1 \%)$ & $86(31.9 \%)$ & \\
\hline
\end{tabular}

On the contrary, risk stratification according to CTS5 was shown to be predictive of the response to the extended endocrine therapy (Fig. 4). In particular, the risk of developing a late distant recurrence with extended endocrine therapy was significantly lower in patients categorized as high-risk
(HR 0.37; 95\% CI 0.15-0.91, $p=0.04$ ) while no significant difference was outlined for low-risk patients (HR 0.03; 95\% CI 0.00-103.29) and intermediate-risk patients (HR 1.09; 95\% CI 0.31-3.89).

Among patients categorized as high risk by CTS5, adjuvant chemotherapy and radiotherapy were comparable both in the extended and in the standard ET groups. The majority of the patients prescribed an extended endocrine regimen underwent surgery after 2005 (Table 4), but no differences in terms of LDR risk could be outlined when compared to patients who underwent an extended ET after surgery performed before that year (HR 0.86, 95\% CI 0.09-8.18) (Figs. 8, 9).

\section{Discussion}

A metanalysis published by Pan $\mathrm{H}$. et al. including more than 60,000 women has shown that the risk of distant recurrence persists for at least 20 years from the diagnosis of an ER+breast cancer [14]. There is now evidence that this risk can be reduced by extending the course of endocrine therapy, but a significant benefit is unlikely to happen to all individual patients.

Many studies have been conducted on this topic: eight RCTs involving more than 17,000 patients were recently included in a metanalysis by Corona et al., which confirmed the beneficial effect of the extended endocrine therapy on DFS but not on OS [15]. The protection offered by the extended endocrine therapy comes along with an increased incidence of therapy-related side effects: endometrial cancer and thromboembolic disease for Tamoxifen, cardiovascular events, and bone fractures for AIs [16]. These results raise several questions when it comes to recommending extended adjuvant endocrine therapy, such as those concerning patient selection, the agent of choice, and the duration of treatment.
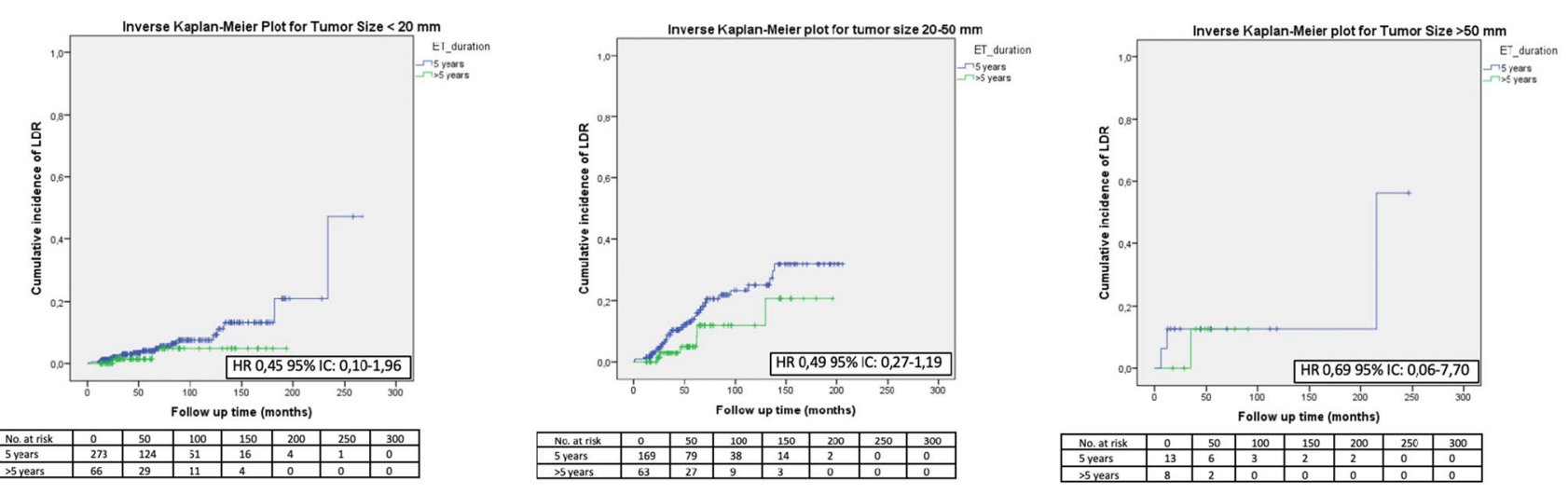

Fig. 1 LDR risk according to ET duration and tumor size 

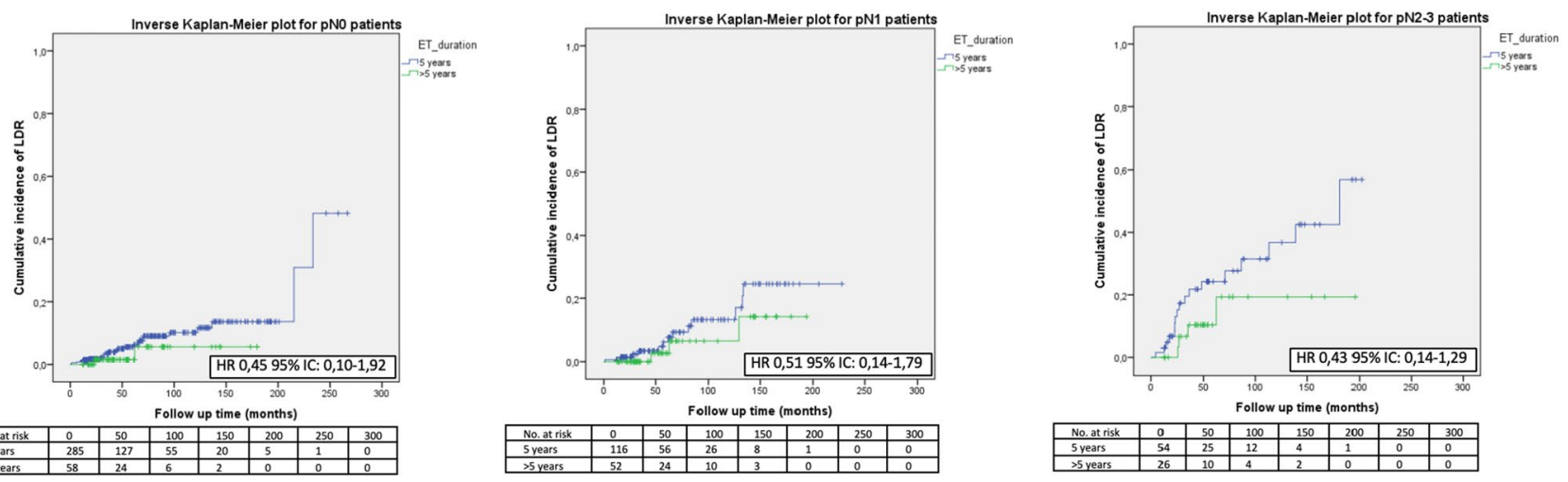

Fig. 2 LDR risk according to ET duration and nodal status
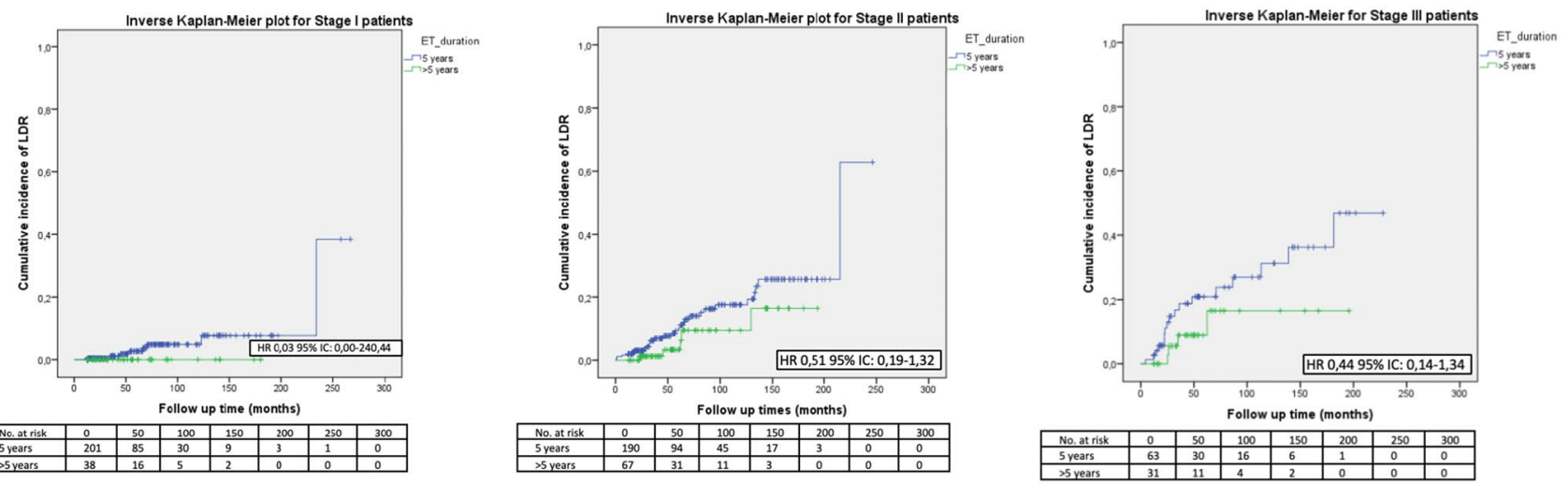

Fig. 3 LDR risk according to ET duration and stage of disease
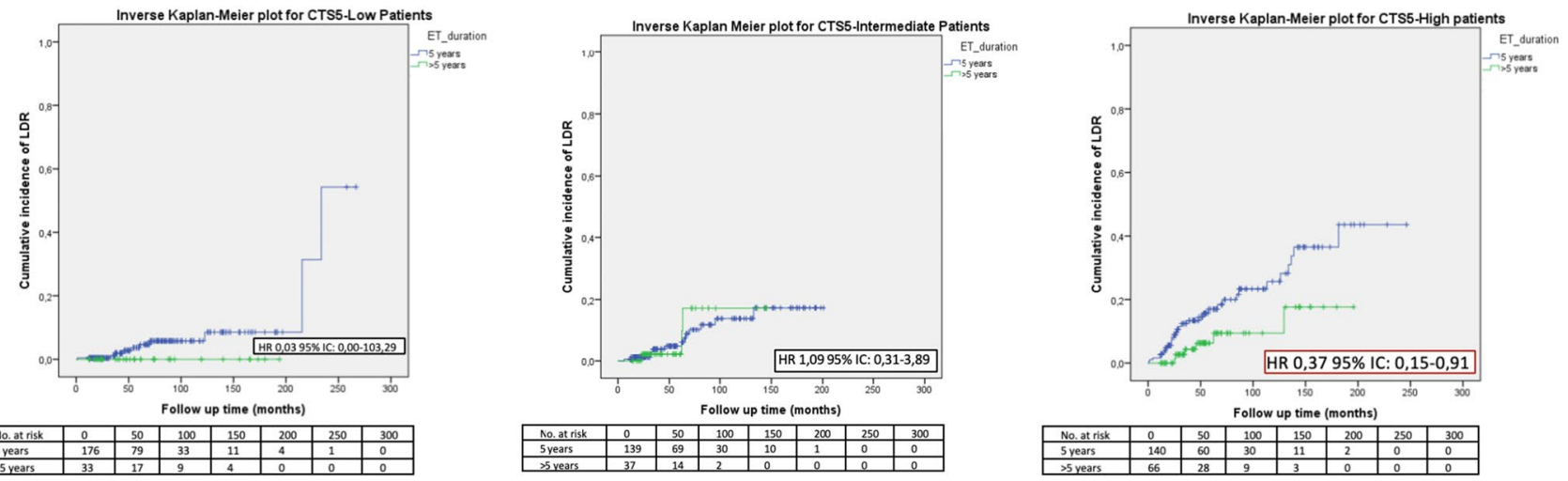

Fig. 4 LDR risk according to ET duration and CTS5

Several multigene assays have been proven to provide prognostic information on the risk of LDR. A recently published pan-genomic analysis by the TransATAC study group compared the performance of six prognostic signatures: CTS, IHC4, BCI prognostic score, Oncotype Dx,
Prosigna, and EndoPredict. While all tools were able to provide prognostic information on the risk of early recurrence, only the BCI score, Prosigna, and EndoPredict demonstrated a significant ability to stratify patients for LDR risk, above and beyond clinical parameters [17]. The 

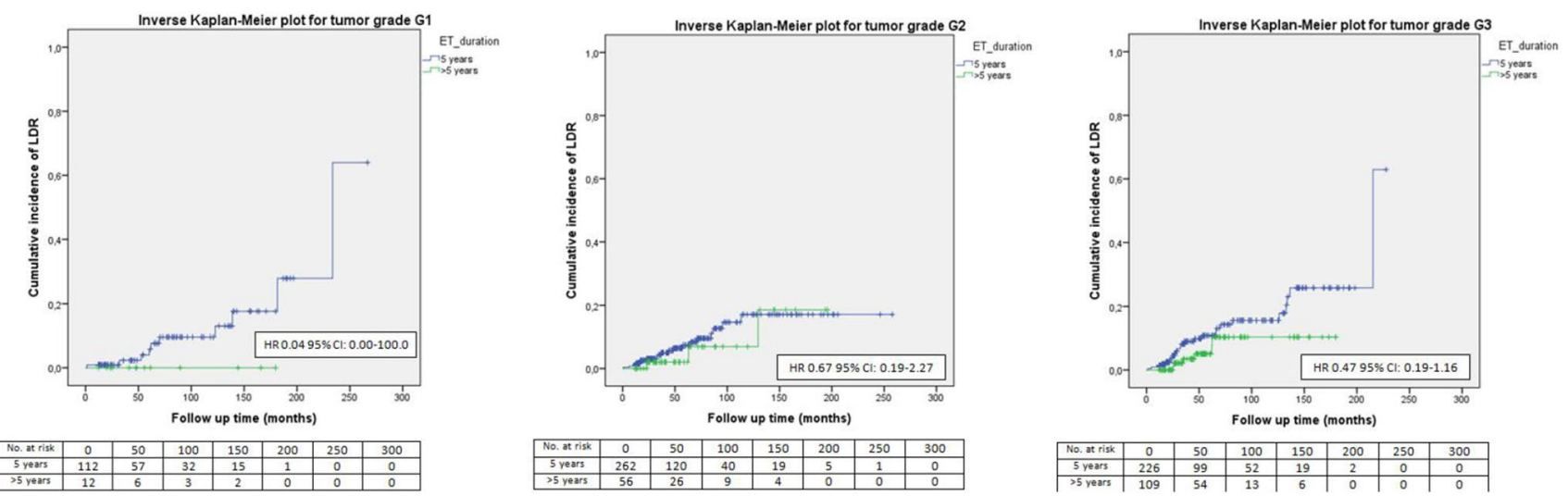

Fig. 5 LDR risk according to ET duration and tumor grade

Fig. 6 LDR risk according to ET duration and Ki67

Fig. 7 LDR risk according to ET duration and PgR status

prognostic performance was weaker among patients with nodal involvement, once more underlying the importance of this clinical variable also in the long term.

So far, little is known about the magnitude of the benefit associated with the extended endocrine treatment according to the risk stratification carried out with the prognostic tools.
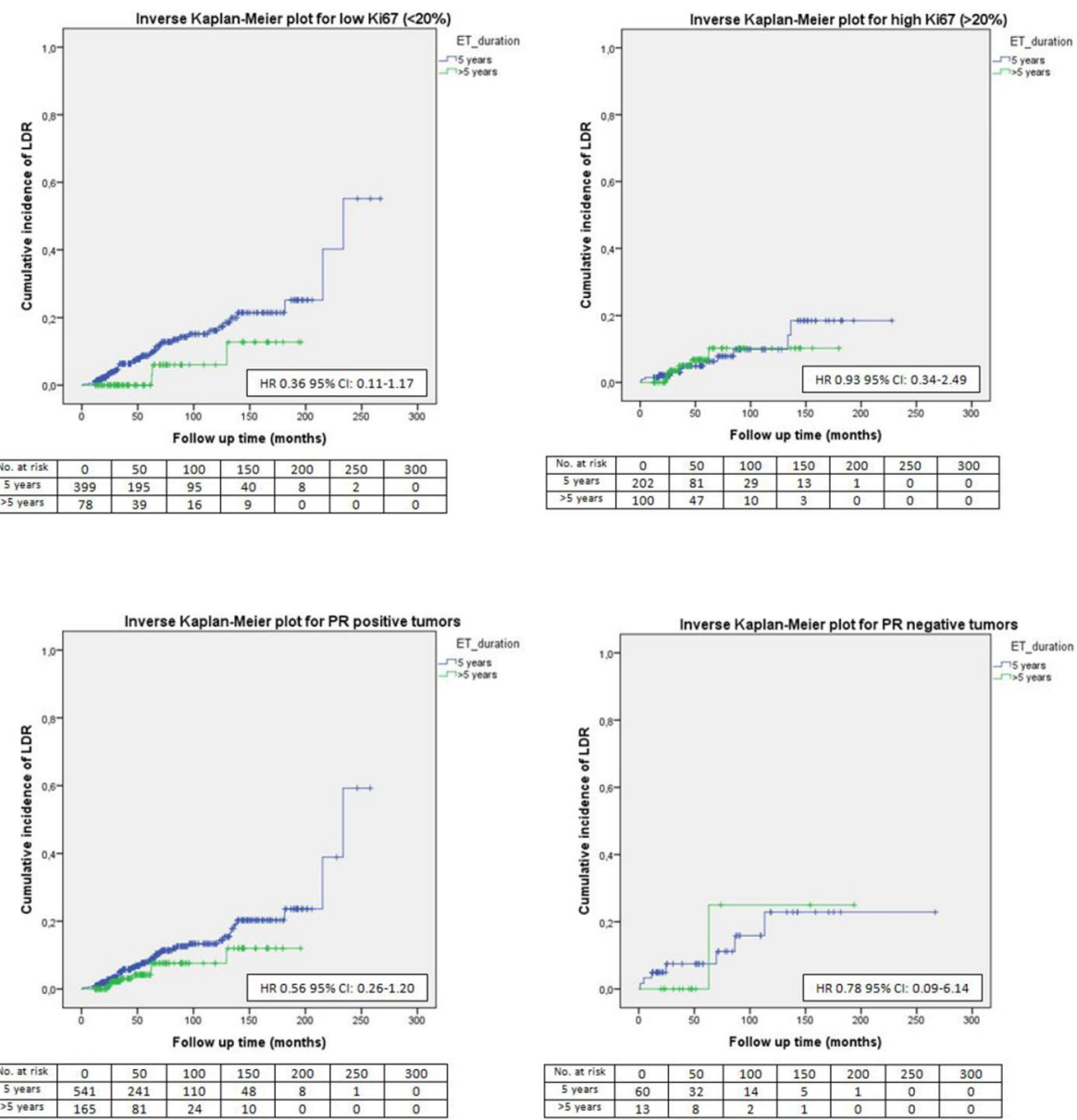

Historically, the decision to propose an extended endocrine regimen was based on the only two clinical variables shown to be independently associated with the risk of late recurrence: tumor size and lymph node burden [18]. More recently, an algorithm integrating four clinicopathological variables, the Clinical Treatment Score at 5 years (CTS5), 
Table 4 CTS5 high risk patients

\begin{tabular}{lrlr}
\hline & 5 years of ET & Extended ET & $p$ value \\
\hline Chemotherapy & & & \\
Yes & $141(65.9 \%)$ & $73(34.1 \%)$ & 0.14 \\
No & $43(76.8 \%)$ & $13(23.2 \%)$ & \\
Radiotherapy & & & \\
Yes & $134(66.7 \%)$ & $67(33.3 \%)$ & 0.45 \\
No & $50(72.5 \%)$ & $19(27.5 \%)$ & \\
Timeframe & & & \\
$\leq 2005$ & $87(84.5 \%)$ & $16(15.5 \%)$ & $<0.001$ \\
$\geq 2006$ & $97(58.1 \%)$ & $70(41.9 \%)$ & \\
\hline
\end{tabular}

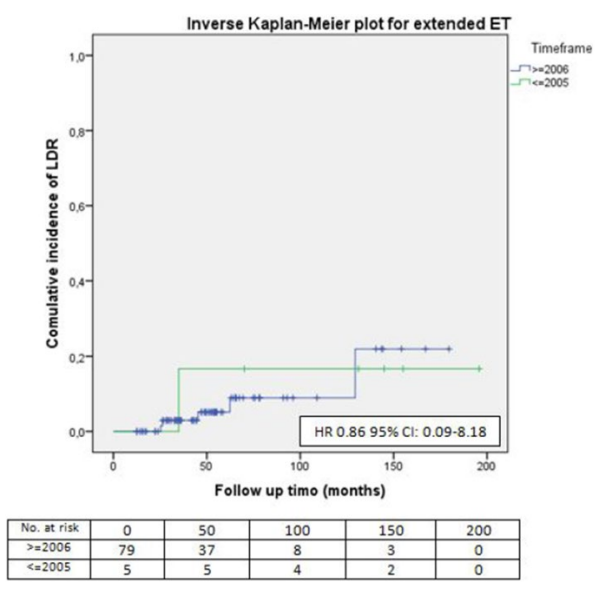

Fig. 8 LDR risk for CTS5 high-risk patients who underwent extended ET according to time of surgery

was shown to be prognostic for late distant recurrence, thus providing useful information to help to tailor the prescription of extended ET. This study was aimed at investigating whether the CTS5-based risk stratification could be helpful in choosing patients more likely to benefit from extended endocrine therapy. The analysis was conducted on a retrospective cohort of 783 patients, of which 180 were prescribed extended endocrine therapy. We found that neither tumor size nor nodal involvement, stage of the disease, tumor grade, Ki67 level, and progesterone receptor status could individually predict the response to such treatment. On the contrary, the risk stratification according to CTS5-based showed a significant benefit of the extended endocrine therapy in high-risk patients with a $60 \%$ reduction in the risk of developing an LDR. This suggests that the CTS5 can help in selecting the patients more likely to profit from the treatment. No benefit from extended ET administration has been observed in low and intermediaterisk patients. It is to be noted that this result was obtained with a median duration of extended endocrine therapy of only 7 years, while the standard consensus considers 10 years of endocrine therapy the ideal objective [19]. It has recently been observed that recent advantages in diagnostics and treatment could have concurred to the better outcomes of the patients treated with extended endocrine therapy [20]; however, we did not observe different LDR risks confronting patients who underwent an extended endocrine therapy after surgery performed before versus after 2005. To our knowledge, this is the first study to evaluate the predictive ability of the CTS5. Only two other prospective-retrospective studies, both involving a component of Breast Cancer Index prognostic score (BCI $\mathrm{H} / \mathrm{I})$, have conducted a similar analysis. BCI (H/I) predictive ability was initially demonstrated in the NCIC-CTG MA.17 RCT cohort $(N=249,60 \% \mathrm{~N}+)$. Patients categorized as BCI (H/I)-High had a significantly improved outcome with extended letrozole treatment versus placebo: a $67 \%$ reduction in risk of recurrence (OR 0.35; 95\% CI 0.16-0.75; $p=0.007$ ), while patients with $\mathrm{BCI}(\mathrm{H} / \mathrm{I})$-low did not have a statistically significant decrease in late recurrence when treated with extended endocrine therapy (OR 0.68 ; 95\% CI $0.31-1.52$; $p=0.35$ ). More recently, Bartlett and Sgroi tested the BCI prognostic index on 583 node-positive aTTom patients. They found a significant benefit from extended tamoxifen for patients classified as BCI (H/I)-High (HR 0.35; 95\% CI $0.15-0.86$ ): The risk of recurrence was $27.0 \%$ and $37.2 \%$ for patients treated with 10- and 5-year tamoxifen, respectively, demonstrating a significant absolute $10.2 \%$ reduction of the risk of recurrence. In contrast, there was no significant benefit from an additional 5 years of tamoxifen in patients classified as BCI (H/I)-Low (HR 1.07; 95\% CI 0.69-1.65). As described by Simon et al., level 1B classification for the clinical utility of a biomarker requires reproducibility of the results in at least two independents prospective-retrospective studies. BCI (H/I) can thus be considered a biomarker of response to extended endocrine therapy. Despite these encouraging results, the routine use of such assays is limited by availability and prohibitive costs. They are not yet recommended for guiding therapy of $\mathrm{ER}+\mathrm{BC}$ beyond 5 years from diagnosis $[21,22]$.

The results of our study suggest that also CTS5 can predict the response to extended endocrine therapy and should hence be taken into account in settings with difficult access to genomic signatures.

Limitations of this study are its pure retrospective design, the small number of patients prescribed the extended endocrine therapy, and the relatively short median follow-up that could have missed some LDR which are yet to develop. Moreover, the prescription of an extended endocrine regimen was based on the clinicians' choice after the assessment of the risk factors taken singularly, since no integration tool was available in the period considered. Hence, to correctly assess the predictive ability of CTS5, we recommend testing the score on a wider cohort until a prospective analysis can be carried out. The point of strength is the inclusion of pre and postmenopausal patients, the majority of whom have been treated 
Fig. 9 Patients selection process

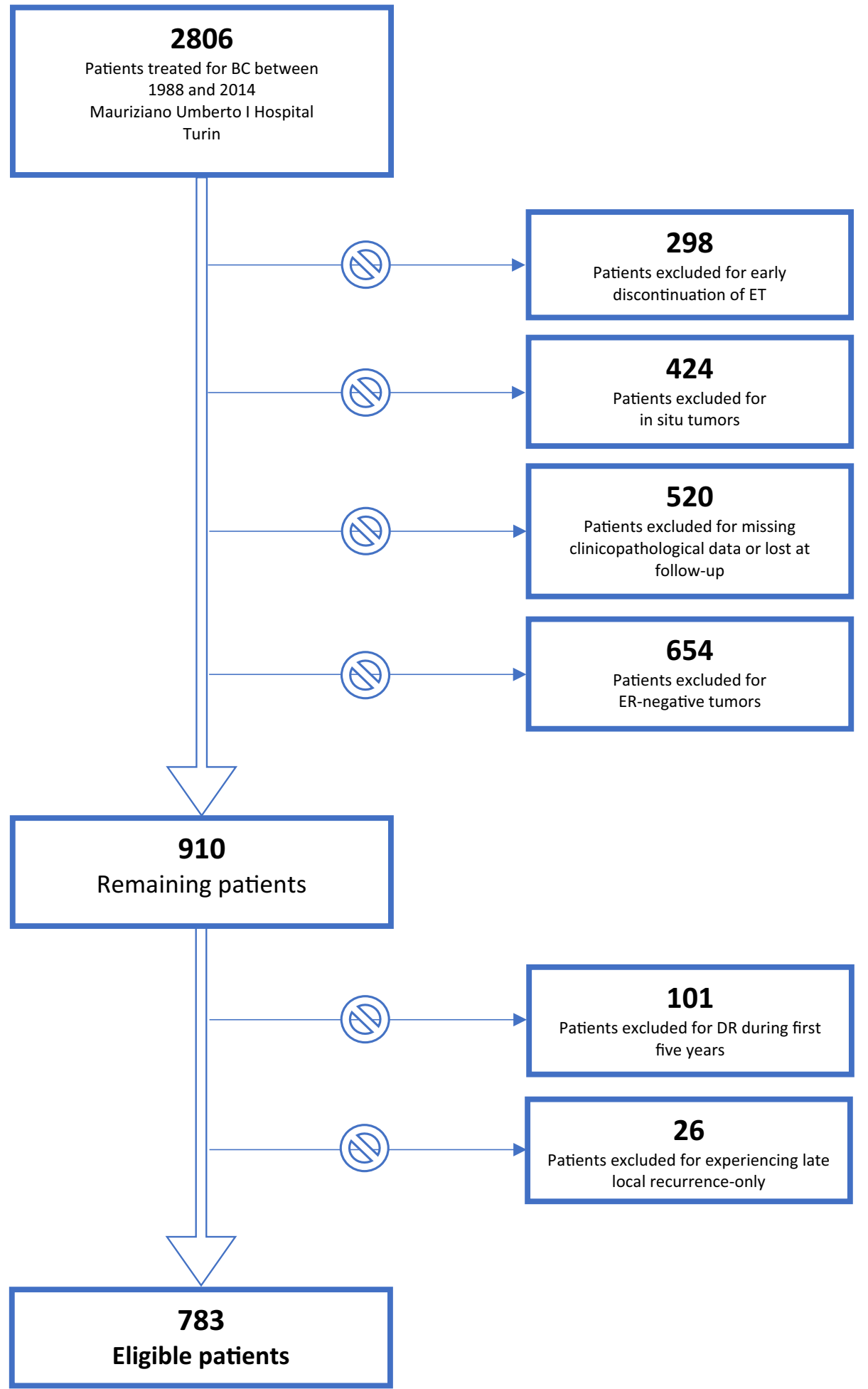


according to current guidelines, an aspect that enhances the applicability of the results of this study to everyday clinical practice.

\section{Conclusions}

Retrospective risk stratification according to CTS5 appeared to be predictive of the response to extended endocrine therapy in our population of real-life pre and postmenopausal patients. Our results suggest its use in the clinical practice to better tailor the prescription of the extended adjuvant endocrine regimen.

Acknowledgements The authors would like to thank Dr. Roberta Rosso for her spirit of abnegation, which allowed to bring this work to completion even during this difficult pandemic period.

Funding Open access funding provided by Università degli Studi di Torino within the CRUI-CARE Agreement. This research did not receive any specific grant from funding agencies in the public, commercial, or not-for-profit sectors.

\section{Declarations}

Conflict of interest Andrea Villasco declares that he has no conflict of interest; Francesca Accomasso declares that she has no conflict of interest; Marta D'Alonzo declares that she has no conflict of interest; Francesca Agnelli declares that she has no conflict of interest; Piero Sismondi declares that he has no conflict of interest; Nicoletta Biglia declares that she has no conflict of interest.

Ethical approval All procedures performed in studies involving human participants were in accordance with the ethical standards of the institutional and national research committee and with the 1964 Helsinki declaration and its later amendments or comparable ethical standards.

Informed consent Informed consent was obtained from all individual participants included in the study.

Open Access This article is licensed under a Creative Commons Attribution 4.0 International License, which permits use, sharing, adaptation, distribution and reproduction in any medium or format, as long as you give appropriate credit to the original author(s) and the source, provide a link to the Creative Commons licence, and indicate if changes were made. The images or other third party material in this article are included in the article's Creative Commons licence, unless indicated otherwise in a credit line to the material. If material is not included in the article's Creative Commons licence and your intended use is not permitted by statutory regulation or exceeds the permitted use, you will need to obtain permission directly from the copyright holder. To view a copy of this licence, visit http://creativecommons.org/licenses/by/4.0/.

\section{References}

1. Henry NL, Shah PD, Haider I, Freer PE, Jagsi R, Sabel MS. Chapter 88: cancer of the breast. In: Niederhuber JE, Armitage JO, Doroshow JH, Kastan MB, Tepper JE, editors. Abeloff's Clinical Oncology, 6th edn. Philadelphia: Elsevier; 2020.
2. American Cancer Society. Cancer Facts \& Figures 2020. Atlanta: American Cancer Society; 2020.

3. Early Breast Cancer Trialists' Collaborative Group (EBCTCG). Aromatase inhibitors versus tamoxifen in early breast cancer: patient-level meta-analysis of the randomised trials. Lancet. 2015;386(10001):1341-52.

4. NCCN Guidelines Version 2. 2020 Invasive Breast Cancer. https:// www.nccn.org/professionals/physician_gls/pdf/breast.pdf. Accessed 13 Feb 2020.

5. Francis PA, Regan MM, Fleming GF, et al. Adjuvant ovarian suppression in premenopausal breast cancer. N Engl J Med. 2015;372:1673.

6. Pagani O, Regan MM, Walley BA, et al. Adjuvant exemestane with ovarian suppression in premenopausal breast cancer. N Engl J Med. 2014;371:107-18.

7. Filipits M, Dubsky P, Rudas M, Greil R, Balic M, Bago-Horvath Z, Singer CF, Hlauschek D, Brown K, Bernhisel R, Kronenwett R, Lancaster JM, Fitzal F, Gnant M. Prediction of distant recurrence using EndoPredict among women with ER(+), HER2(-) node-positive and node-negative breast cancer treated with endocrine therapy only. Clin Cancer Res. 2019;25(13):3865-72.

8. Dowsett M, Sestak I, Regan MM, Dodson A, Viale G, Thürlimann B, Colleoni M, Cuzick J. Integration of clinical variables for the prediction of late distant recurrence in patients with estrogen receptorpositive breast Cancer treated with 5 years of endocrine therapy: CTS5. J Clin Oncol. 2018;36(19):1941-8.

9. Villasco A, Agnelli F, D’Alonzo M, Accomasso F, Sismondi P, Biglia N. Validation of CTS5 on a retrospective cohort of real-life pre- and postmenopausal patients diagnosed with estrogen receptor-positive breast cancers: Is it prognostic? Clin Breast Cancer. 2020;21(20):S1526-8209.

10. Richman J, Ring AE, Dowsett M, Sestak I. Clinical validity of CTS5 for estimating risk of late recurrence in unselected, non-trial patients with early ER+ breast cancer. J Clin Oncol. 2019;37(15_suppl):514.

11. Lee J, Cha C, Ahn SG, Kim D, Park S, Bae SJ, Kim J, Park HS, Park S, Kim SI, Park BW, Jeong J. Validation of Clinical Treatment Score post-5 years (CTS5) risk stratification in premenopausal breast cancer patients and Ki-67 labelling index. Sci Rep. 2020;10(1):16850. https://doi.org/10.1038/s41598-020-74055-3. Erratum in: Sci Rep. 2021;11(1):5415.

12. Wang C, Chen C, Lin Y, Zhou Y, Mao F, Zhu H, Zhang X, Shen S, Huang X, Wang X, Zhao B, Yang J, Sun Q. Validation of CTS5 model in large-scale breast cancer population and the impact of menopausal and HER2 status on its prognostic value. Sci Rep. 2020;10(1):4660.

13. Sgroi DC, Carney E, Zarrella E, Steffel L, Binns SN, Finkelstein DM, Szymonifka J, Bhan AK, Shepherd LE, Zhang Y, Schnabel CA, Erlander MG, Ingle JN, Porter P, Muss HB, Pritchard KI, Tu D, Rimm DL, Goss PE. Prediction of late disease recurrence and extended adjuvant letrozole benefit by the HOXB13/IL17BR biomarker. J Natl Cancer Inst. 2013;105(14):1036-42.

14. Bartlett JMS, Sgroi DC, Treuner K, Zhang Y, Ahmed I, Piper T, Salunga R, Brachtel EF, Pirrie SJ, Schnabel CA, Rea DW. Breast Cancer Index and prediction of benefit from extended endocrine therapy in breast cancer patients treated in the adjuvant TamoxifenTo Offer More? (aTTom) trial. Ann Oncol. 2019;30(11):1776-83.

15. Pan H, Gray R, Braybrooke J, Davies C, Taylor C, McGale P, Peto R, Pritchard KI, Bergh J, Dowsett M, Hayes DF, EBCTCG. 20-year risks of breast-cancer recurrence after stopping endocrine therapy at 5 years. N Engl J Med. 2017;377(19):1836-46.

16. Corona SP, Roviello G, Strina C, Milani M, Madaro S, Zanoni D, Allevi G, Aguggini S, Cappelletti MR, Francaviglia M, Azzini C, Cocconi A, Sirico M, Bortul M, Zanconati F, Giudici F, Rosellini P, Meani F, Pagani O, Generali D. Efficacy of extended aromatase inhibitors for hormone-receptor-positive breast 
cancer: a literature-based meta-analysis of randomized trials. Breast. 2019;46:19-24.

17. Goldvaser H, Barnes TA, Šeruga B, Cescon DW, Ocaña A, Ribnikar D, Amir E. Toxicity of extended adjuvant therapy with aromatase inhibitors in early breast cancer: a systematic review and meta-analysis. J Natl Cancer Inst. 2018;110(1):31-9.

18. Sestak I, Buus R, Cuzick J, Dubsky P, Kronenwett R, Denkert C, Ferree S, Sgroi D, Schnabel C, Baehner FL, Mallon E, Dowsett M. Comparison of the performance of 6 prognostic signatures for estrogen receptor-positive breast cancer: a secondary analysis of a randomized clinical trial. JAMA Oncol. 2018;4(4):545-53.

19. Lee ES, et al. Factors associated with late recurrence after completion of 5-year adjuvant tamoxifen in estrogen receptor positive breast cancer. BMC Cancer. 2016;16:430.

20. Burstein HJ, Curigliano G, Loibl S, Dubsky P, Gnant M, Poortmans P, Colleoni M, Denkert C, Piccart-Gebhart M, Regan M, Senn HJ, Winer EP, Thurlimann B; Members of the St. Gallen International Consensus Panel on the Primary Therapy of Early Breast Cancer 2019. Estimating the benefits of therapy for early-stage breast cancer: the St. Gallen International Consensus Guidelines for the primary therapy of early breast cancer 2019. Ann Oncol. 2019;30(10):1541-57.

21. Noordhoek I, Blok EJ, Meershoek-Klein Kranenbarg E, Putter H, Duijm-de Carpentier M, Rutgers EJT, Seynaeve C, Bartlett JMS, Vannetzel JM, Rea DW, Hasenburg A, Paridaens R, Markopoulos CJ, Hozumi Y, Portielje JEA, Kroep JR, van de Velde CJH, Liefers GJ. Overestimation of late distant recurrences in high-risk patients with ER-positive breast cancer: validity and accuracy of the CTS5 risk score in the TEAM and IDEAL trials. J Clin Oncol. 2020;38(28):3273-81.

22. Rossi L, McCartney A, De Santo I, Risi E, Moretti E, Malorni L, Biganzoli L, Di Leo A. The optimal duration of adjuvant endocrine therapy in early luminal breast cancer: a concise review. Cancer Treat Rev. 2019;74:29-34.

Publisher's Note Springer Nature remains neutral with regard to jurisdictional claims in published maps and institutional affiliations. 